\title{
Event Detection in Multiple Webpages based on Comprehensive Dimension Matching and Co-occurrence Constraint
}

\author{
Yuanzi Xu, Qingzhong $\mathrm{Li}^{*}$, Zhongmin Yan and Wei Wang \\ School of Computer Science and Technology, Shandong University, Jinan, 250101, China
}

Received: 12 Jun. 2013, Revised: 17 Oct. 2013, Accepted: 18 Oct. 2013

Published online: 1 May. 2014

\begin{abstract}
Detecting various sentence-level events from multiple webpages can be important in finding knowledge. We propose an event detection method based on comprehensive dimension matching and co-occurrence constraint. First, we detect events from a single webpage by clustering co-reference sentence-level event mentions. These events are considered as co-occurrence events in every single webpage. Second, similar events from multiple webpages are clustered. The dimension matching method is used to aggregate event mentions. Different matchers measure different dimensions, and an extended evidence theory is proposed to allocate dynamic weight and combine dimension measurement results. We propose an event co-occurrence constraint to reduce match times and quantity of candidate matches events in the multiple webpages event-detection process to improve event cluster efficiency. The experiment results demonstrate that this method can detect various events and noticeably reduce the quantity of co-reference events.
\end{abstract}

Keywords: Event detection, comprehensive dimension matching, co-occurrence constraint, extended evidence theory

\section{Introduction}

Event detection is the task of identifying events in news. Most of events which are reported in webpages are unstructured data. Information management not only integrates structured data such as entity attributes and relationships between entities in the Web but also integrates events. Existed event detection approaches are inapplicable for discovering events that are participated by specific entity and the type of them cannot be predefined. Examples of such events include enterprise activities in the news. Integrating multiple enterprise webpages and detecting valuable entity events may help enterprise policymakers understand themselves and the development of novel trends in other enterprises, as well as improve market intelligence. An event is an activity that occurs at a special time and involves participants. An event mention is the sentence in which an event is reported. The descriptions of the same event from different webpages are inconsistent. These event mentions that report the same event are co-reference event mentions. If each event mention is considered an event, many duplicate events will occur. Thus, clustering co-reference event mentions as an event can reduce the number of duplicate events. Our goal is to detect events in multiple webpages by clustering co-reference event mentions. This approach is important to provide valuable market information to enterprises.

Detecting such events in multiple webpages poses several interesting technical challenges. First, event mentions are unstructured data, and the direct use of attribute similarity to judge entity attributes is difficult. For example, Fig. 1 depicts that an event has four co-reference event mentions. However, the texts of these co-reference event mentions have obvious differences. We use several dimensions to express event mentions and use comprehensive matchers to measure the similarities of these dimensions. Second, we find some events in single webpage by clustering co-reference event mentions. These events have obvious differences and can be considered as co-occurrence events. Co-occurrence events do not need to calculate the similarity between them and they can be used to reduce event match times in following event detection. Third, clustering event mentions in multiple webpages require considerable running time. We

\footnotetext{
*Corresponding author e-mail: lqz@sdu.edu.cn
} 


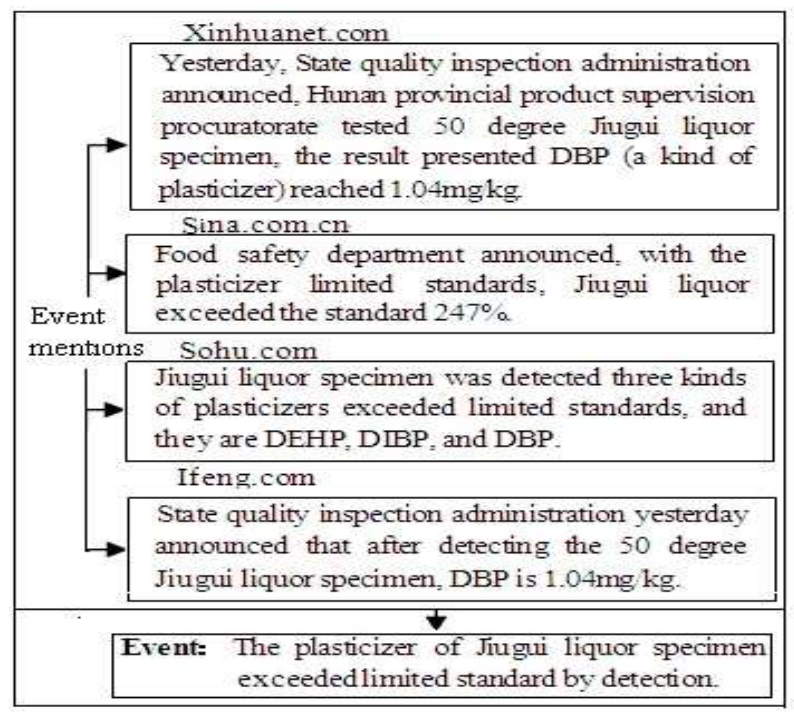

Fig. 1: Four co-reference event mentions for same event

propose the two-stage event detection method and the use of a constraint to reduce the running time.

Fig. 1 illustrates an event that has four co-reference event mentions. The event mention in Xinhuanet.com reports the plasticizer name. The event mention in Sina.com.cn reports the percentage of plasticizer that exceeds the limit standard. The event mention in Sohu.com introduces the three plasticizers. These co-reference event mentions are extracted from different webpages in the same day. However, these co-reference event mentions only use text similarities cannot find references to the same event. To improve event cluster accuracy, we adopt frame semantics [1,2] and use dimensions such as time, subject, object, and activity to represent event mention. First of all, we measure the similarity of each dimension. Thereafter, we use extended evidence theory to combine the similarities of key dimensions as the similarity of the final event mention. Considering that composite dimension matching can maximize the use of syntactic structure information and semantic information, this method can increase the recall and precision ratio of event detection.

We present a two-stage sentence-level event detection method, i.e., EDCoAGENES, to cluster co-reference event mentions in multiple webpages. Given a time period and a set of entities, we label event mentions that contain target entities in every webpage. In a single webpage, we cluster co-reference event mentions and find co-occurrence events. In multiple webpages, we use co-occurrence constraint to reduce match times and quantity of candidate matches events and we cluster similar events to detect many different events. By using the EDCoAGENES method, we not only detect various events but also can understand the event burst degree during a particular time period according to the amount of co-reference event mentions.

In summary, we introduce a two-stage sentence level event detection method to discover multiple webpage events. In this method, the second stage can use the co-occurrence events which are found in the first stage to cluster events. We use several dimensions to present event mentions and use comprehensive matchers in measuring event mentions to improve cluster accuracy. In this paper, we propose the use of event co-occurrence constraint to restrict the event detection of multiple webpages and reduce the running time during the cluster process.

This paper is organized as follows. We briefly review some related research efforts in Section 2, and describe the problem in Section 3.The proposed approach is introduced in Section 4 and the experimental evaluations are reported in Section 5. In the last section, we draw conclusions.

\section{Research Background}

In recent years, event detection research has increasingly focused on large-scale webpages and has become the most popular research in information management [3], data integration, information retrieval, and artificial intelligence. Information retrieval identifies similar changes in the relationship [4] between certain entities, such as simultaneously rise and decline, to detect events. Social tagging [5,6] discovers burst tags in the same period to detect a few special events. Manoj [7] found real-time events according to high frequency words in micro-blogs reported by the same author. Detecting many retrospective events in multiple webpages is important for finding knowledge. Event detection research involves single webpage detection and multiple webpages detection.

In single webpage event detection, Yan [8] found paragraph boundary events and considered them interrelated and independent. We propose the event co-occurrence constraint which is based on this idea of the independent relationship between events. In multiple webpage event detection, some studies have adopted predefined-type event detection. Shan [9] used event keywords to detect burst events. Zhao [10] appended time characteristics to event keywords, such as "2008 US presidential election", which detects time-characteristic burst events. Given that detailed event information is needed to mine sentences in a webpage, current research has now focused on sentence-level event detection. Martina [11] proposed sentence-level event detection in news webpages and found predefined type events. Jiang [12] used open information extraction and proposed event ontology to find various events. David [13] thought important named entities, such as time, place, people, and organization, and the co-occurrence relationships between them were also used to find events. Cluster method is important for event detection. Compared with another event cluster method [14,15], the agglomerative 
hierarchical cluster method [16] possesses certain advantages, such as accurate differentiation of objects, automatic determination of the number of clusters, and discovery of arbitrary clustering shapes. Thus, the agglomerative hierarchical cluster method is suitable for event detection.

Considering that many co-reference event mentions have missing and inconsistent values, the clustering of sentence-level event mentions according only to text similarity judgment exhibits low accuracy. Topic detection and tracking research (TDT) [17] uses the TFIDF method to calculate event keywords and uses keyword similarity as event similarity. Given that the same keywords in different syntactic structures, such as subject or object, can express different events, the use of syntactic structure can improve cluster accuracy. Li [18] demonstrated that comprehensive similarity can improve clustering precision for complex data structures. Evidence theory [19] combines several dimension similarities with static weight to provide a uniform result.

Our research used eight dimensions to present event mentions and combined syntactic structures and keyword semantic meanings to measure event mention similarity. We extended the evidence theory to allocate dynamic weights to different dimensions according to the ability of such dimensions to provide similarity.

\section{Event Mention and Event}

To present and facilitate the following discussions clearly, we explain some concepts used in this paper in this section.

Event mention. An event mention is a sentence in which an event is reported. An event can have many mentions that refer to it. In this paper we use eight dimensions to represent event mentions according to the definition of an event. The eight dimensions are denoted as $\{$ agent, activity, $\{$ object $\}$, time, $\{$ location $\},\{$ cause \}$,\{$ purpose $\},\{$ manner $\}$. The event mention set is represented as $E M=\left\{e m_{11}, e m_{12}, \cdots, e m_{i 1}, \cdots, e m_{i j}\right.$, $\left.\cdots, e m_{n k}\right\}$, and $e m_{i j}(1 \leq i \leq n, 1 \leq j \leq k)$ represents an event mention in event $e_{i}$.

Event. An event is an entity activity that occurs at a specific time and place. An event is constructed by certain elements, such as time, agent, activity, object, location, cause, purpose, and manner. An event set is indicated as $E=\left\{e_{1}, e_{2}, \cdots, e_{n}\right\}$, and an event is expressed as $e_{i}(1 \leq i \leq n)$.

A data source provides event mentions, such as web sites, databases, etc. A set of data sources can be represented as $S=\left\{s_{1}, s_{2}, \cdots, s_{n}\right\}$.Some event mentions can point to the same event or to different events. The relationship between source, document, event mention, and event is shown in Fig 2.

Event Detection. Event detection is the automatic identification and classification of co-reference event mentions to find various events in mutiple webpages. Event detection is divided into single webpage event
Source Document Event Mention Event

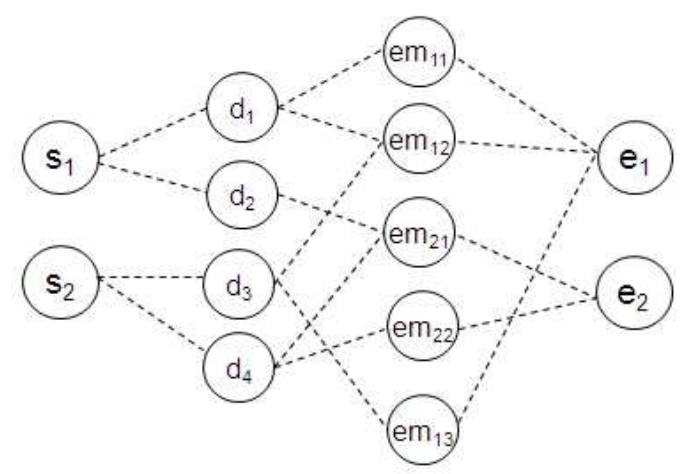

Fig. 2: Source, document, event mention, event, and their relationships

detection and mutiple webpages event detecion. Detected events from a single webpage construct co-occurrence events and they can simplify event detection in mutiple webpages. Event $e_{i}$ can be represented as $e_{i}=\left\{e m_{i 1}, e m_{i 2}, \cdots, e m_{i n}\right\}$, and event set can be represented as $E=\left\{e_{1}, e_{2}, \cdots, e_{i}\right\}=$ $\left\{\left\{e m_{11}, e m_{12}, \cdots, e m_{1 k}\right\}, \cdots,\left\{e m_{i 1}, e m_{i 2}, \cdots, e m_{i n}\right\}\right\} \cdot k, p$ and $n$ are the number of co-reference event mentions.

Co-occurrence Event. Events clustered from a single webpage are independent events which have obvious differences. Independent events from a single webpage are co-occurrence events. Co-occurrence events need not to calculate similarity in mutiple webpages event detection becuse they are obviously different. For example, if $\left\{e_{1}, e_{2}, \cdots, e_{n}\right\} \subseteq d_{i}$ then $\left\{e_{1}, e_{2}, \cdots, e_{n}\right\}$ constitute co-occurrence event.

Event co-occurrence constraint. The event co-occurrence constraint restricts co-occurrence events to eliminate the need to calculate the similarity between co-occurrence events. Events that are detected in the same webpage constitute co-occurrence events. Co-occurrence events are defined as $E_{d}$. and d is the document in which we find some events. Two events from different webpages constitute an event pair. If the similarity of an event pair reaches the threshold, the event similarity in the matched event pair do not need to be computed with the co-occurrence event similarity.

\section{Event Detection}

\subsection{Event detection process}

In this paper, we present an event detection method named EDCoAGENES based on comprehensive dimension matching and co-occurrence constraints. This method can detect many events and reduce duplicates in event quantity. We use a slide window mechanism 
according to the time period to obtain webpages. The whole event detection process is shown in Fig.3. The three steps involved in the event detection process are introduced as follows.

Step 1. An event mention is labeled in the webpages. The event mention that contains the target entities is then labeled by using related webpages. We use the Chinese word segmentation tool ICTCLAS 3.1 to handle event mention and use eight dimensions to represent the event mention. We filter the content of the webpage, label the event mention, and use dimensions to represent the event mention.

Step 2. An event is detected in a single webpage. In this step, we identify co-reference event mentions and cluster them in a single webpage. These events which are clustered from a single webpage are co-occurrence events. For example, the event mentions $e m_{1}$ and $e m_{2}$ in $D_{1}$ both refer to event $e_{1}$ ( Fig. 3). These event mentions are co-reference event mentions that need to be classified in a cluster. Four event mentions in $D_{1}$ are classified into two clusters, which are referred to as event $e_{1}$ and $e_{2}$. Event $e_{1}$ and $e_{2}$ are co-occurrence events because they are detected in the same webpage. To compute for event mention similarity, we use the comprehensive dimension matching method and extended evidence theory model to allocate dynamic dimension weights. We classify the single webpage co-reference event mentions in a cluster as an event and do not merge them.

Step 3. An event is detected in multiple webpages. We pair any two webpages to cluster similar events and place the resulting cluster in a document. After that, we pair the document with another webpage to cluster similar events. For example, we pair $D_{1}$ and $D_{2}$ to cluster events, place the results in a document, and pair this document with $D_{3}$. In this process we find that $e_{1}$ and $e_{3}$ are similar events, and that $e_{4}$ and $e_{5}$ are similar events. We propose an event co-occurrence constraint to restrict clusters in a webpage pair. Event $e_{1}$ and $e_{2}$ are co-occurrence events in $D_{1}$, and events $e_{3}$ and $e_{4}$ are co-occurrence events in $D_{2}$. In the matching process we find that the similarity of $e_{1}$ and $e_{3}$ reached the threshold. We then aggregate $e_{1}$ and $e_{3}$ and do not compute the similarity of $e_{1}, e_{4}$, and $e_{2}, e_{3}$ because they are subjected to the event co-occurrence constraint.

Fig. 3 shows the three steps of event detection. We use the event detection algorithm to cluster co-reference event mentions in multiple webpages. The core algorithm is presented in Algorithm 1.

Algorithm 1 first initializes event set $E$ and every document $d_{i}$ from set $D$ in Line 1 . Lines 2 to 5 show the process of single webpage event detection. Line 3 labels the event mention $E M_{d}$ from each document $d_{i}$, and Line 4 uses a function to aggregate event mentions if their similarity is greater than $T$. Lines 6 to 8 show event detection in multiple webpages. We pair two event sets to compare event similarity until all event sets are compared. AggregateEvent is a function that clusters similar events from two event sets and will be presented later.

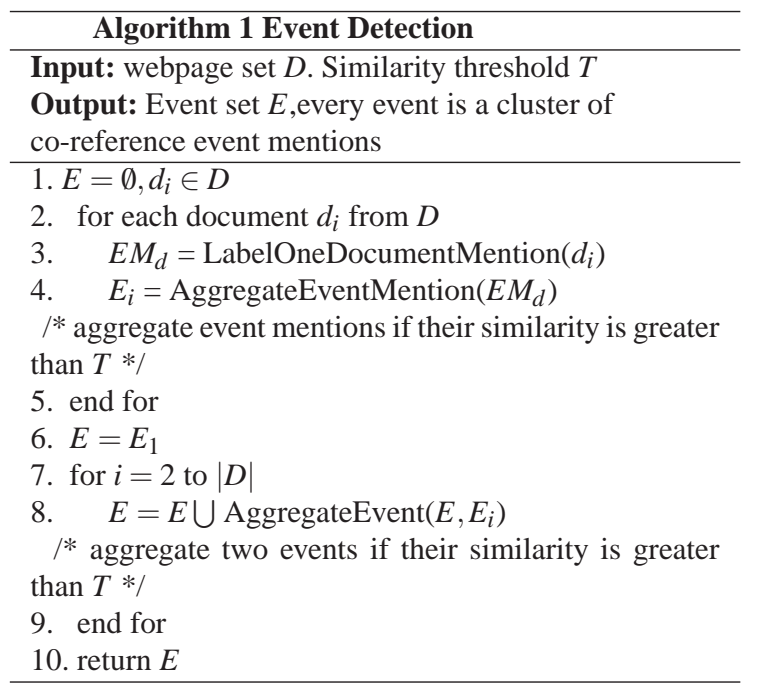

\subsection{Event detection in a single webpage}

Event detection in a single webpage uses the hierarchical clustering method to cluster co-reference event mentions that refer to the same event because co-reference event mentions differ from literal descriptions. In clustering process we use dimension matching method. We use eight dimensions to represent event mentions to combine syntactic structures and text similarities for event mention comparisons. We find that the time, subject, object, and activity dimensions are usually not blank and other dimensions are usually blank or do not conform to other corresponding dimensions. According to event definition, time, activity and participants are key elements in an event. In this paper we divided participants into subject and object by different roles. So time, activity, subject and object are key dimensions which can basically represent an event. In this stage, we choose the time, subject, object, and activity dimensions as key dimensions in comparing event mention similarity.

We select event mentions in different webpages to explain dimension matching in Fig. 4. The time dimension value is the time the event occurred according to when the webpage reported the event and the specific time it appeared in the news. We also use a conjecture event occurrence time by using an offset value from the news, such as yesterday and two days ago. The activity dimension value is an activity verb that is extracted from a phrase by using the shallow semantic parsing method. We calculate the similarity of each dimension, and use extended evidence theory to compute for comprehensive dimension similarity. After clustering co-reference event mentions in a single webpage, we consider events which are detected in the same webpage as co-occurrence events.

In Fig.4, the first three event mentions refer to the same event even though these event mentions have noticeable differences in their literal descriptions. The key dimension 


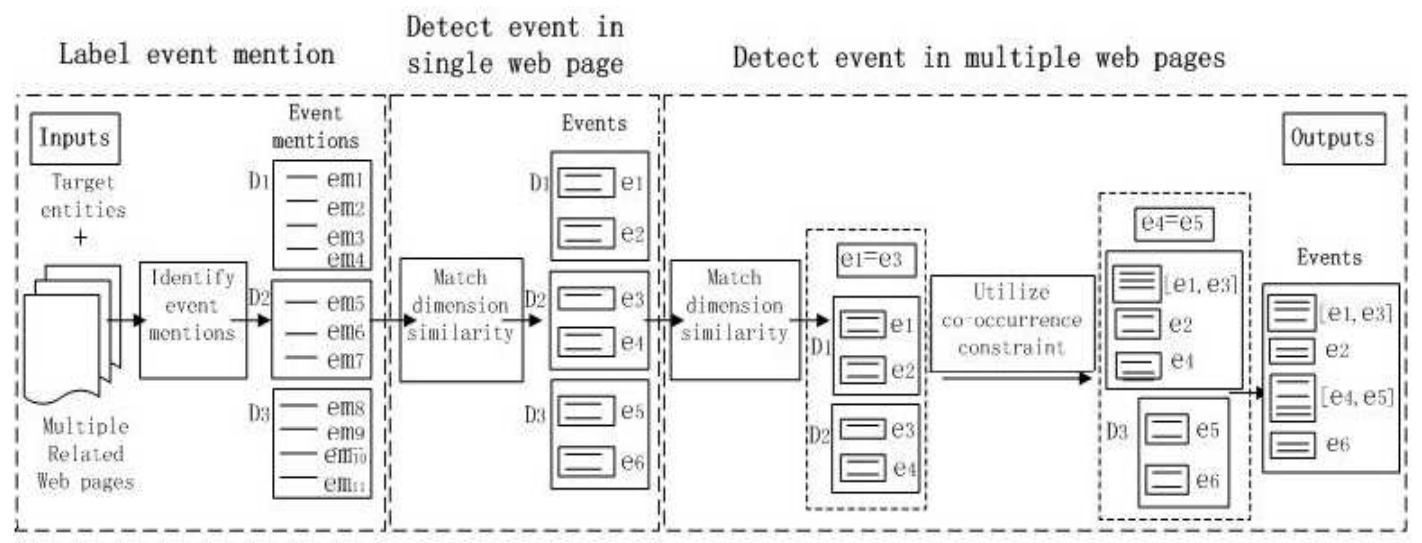

Fig. 3: Event detection process in multiple webpages

\begin{tabular}{|l|l|}
\hline \multicolumn{1}{|c|}{ Event mention } & \multicolumn{1}{|c|}{ Key dimension value } \\
\hline $\begin{array}{l}\text { Food safety department announced, with the } \\
\text { plasticizer limited standards, Jiugui liquor } \\
\text { exceeded the standard } 247 \%\end{array}$ & $\begin{array}{l}2012.11 .19 \mid \text { Jiugui liquor } \\
\text { plasticizers | exceeded }\end{array}$ \\
\hline $\begin{array}{l}\text { Jiugui liquor specimen was detected three kinds } \\
\text { of plasticizers exceeded limited standards, and } \\
\text { they are DEHP, DIBP, and DBP. }\end{array}$ & $\begin{array}{l}2012.11 .19 \mid \text { Jiugui liquor } \mid \\
\text { plasticizers | detected }\end{array}$ \\
\hline $\begin{array}{l}\text { State quality inspection administration yesterday } \\
\text { announced that after detecting the 50 degree } \\
\text { Jiugui liquor specimen, DBP is 1.04mg kg. }\end{array}$ & $\begin{array}{l}2012.11 .19 \mid 50 \text { degree } \\
\text { Jiugui liquor }|\mathrm{DBP}| \\
\text { detecting }\end{array}$ \\
\hline $\begin{array}{l}\text { After Jugui liquor company published the } \\
\text { announcement of plasticizers, stodk (code } 000799) \\
\text { temporary cease trading in November } 19,2012 .\end{array}$ & $\begin{array}{l}2012.11 .19 \mid \text { company } \mid \\
\text { stock } \mid \text { cease trading }\end{array}$ \\
\hline
\end{tabular}

Fig. 4: Key dimensions of event mentions

values of the event mentions show slight differences. The last event mention refers another event, and we can clearly distinguish such an event according to the key dimension. In this paper, we detect events in a short time period (i.e., one week).

\subsection{Event detection in multiple webpages}

Event detection in multiple webpages pairs two webpages and uses the hierarchical clustering method to aggregate similar events. In this paper, we use event co-occurrence constraint to reduce event match times and quantity of candidate matches events in multiple webpages cluster process.

We note that co-occurrence events do not need to calculate similarity according to the constraint. Co-occurrence events can quickly cluster similar events in webpage pairs. We propose two rules to reduce match times and quantity of candidate matches events by using the event co-occurrence constraint.

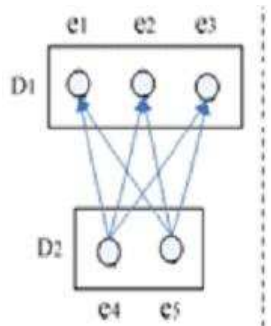

(a) AGENES method

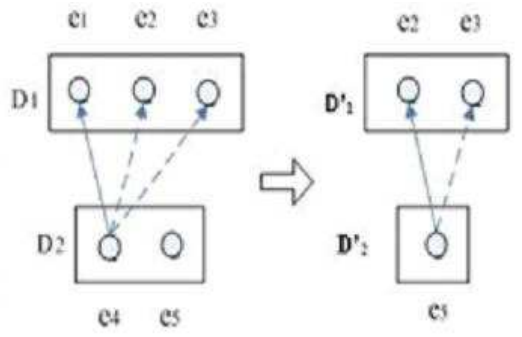

(b) EDCOAGENES method
Fig. 5: Comparisons of co-occurrence constraint

Rule 1. If the similarity of an event pair reaches the threshold, the comparison process can be terminated. For example, if $e_{i} \in E_{1}$ and $e_{j} \in E_{2}$ construct a matched event pair $\left\langle e_{i}, e_{j}\right\rangle$, the comparison process, which searches for an event similar to $e_{i}$, is terminated. Because the candidate matches events in $E_{2}$ are co-occurrence events of $e_{j}$. This rule can be used to reduce match times in event comparison process.

Rule 2. If a matched event pair in two webpages is found, the two events can be deleted from the candidate match event set. For example, for a matched event pair $<e_{i}, e_{j}>, e_{i} \in E_{1}$, and $e_{j} \in E_{2}$, the remainder events in $E_{1}$ are the co-occurrence events with $e_{i}$, and a matched event pair cannot be constructed with $e_{j}$. Thus, the candidate matches event sets are $\left\{E_{2}-e_{j}\right\}$ and $\left\{E_{1}-e_{i}\right\}$. This rule can be used to reduce the number of candidate matches events in event comparison process.

Fig. 5 show the difference in the comparison process of the agglomerative hierarchical clustering method (AGENES) and EDCoAGENES.

Fig. 5(a) shows the process used in the AGENES method if $\left\langle e_{1}, e_{4}\right\rangle$ is the matched event pair that compared $e_{4}$ with the remaining events in $D_{1}$. Fig. 5(b) presents how the EDCoAGENES method is used if the 


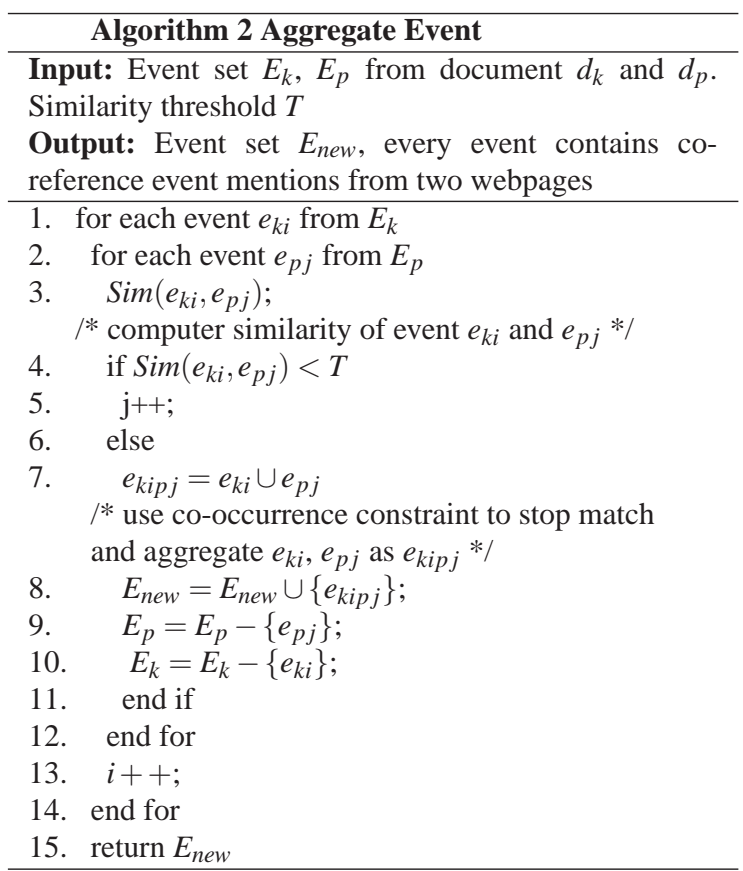

matched event pair is $\left\langle e_{1}, e_{4}\right\rangle$. Comparisons for finding similar events at $e_{4}$ can be stopped. Thereafter, $e_{1}$ from $D_{1}$ and $e_{4}$ from $D_{2}$ are deleted. Dashed arrows point to the unnecessary comparisons in Fig.5(b). Given that $e_{1}, e_{2}$, and $e_{3}$ are co-occurrence events in $D_{1}$, and $e_{4}, e_{5}$ are co-occurrence events in $D_{2}$, we use the constraint only when we need to compare the similarity of $e_{5}$ and $e_{2}$. The left part of Fig.5(b) shows that we can reduce match times of $e_{4}$ by rule1. According to rule2, only $e_{2}$ and $e_{3}$ are candidate matches events of $e_{5}$. Reducing the number of candidate matches events is shown in the right part of Fig.5(b).

Algorithm 2 shows how constraint restricts clustering to aggregate similar events in two webpages.

Lines 1 and 2 circularly compare the events between $E_{k}$ and $E_{p}$. Line 3 computes for the similarity between $e_{k i}$ and $e_{p j}$. If the similarity is below the threshold $T$, the similarity of the next event in $E_{p}$ with $e_{k i}$ is computed. Lines 7 and 8 use an event co-occurrence constraint to aggregate $e_{k i}$ and $e_{p j}$ if their similarity reaches T. Lines 9 and 10 delete $e_{p j}$ from $E_{p}$ and $e_{k i}$ from $E_{k}$ by co-occurrence constraint.

We propose that the use of event co-occurrence constraint reduce running time. We assume that event set $E_{1}$ has $m$ events and event set $E_{2}$ has $n$ events. AGENES compares each event to all events in another set (Fig.5) and the time complexity is $O(m+n)^{2}$. EDCoAGENES finds two events that have reached the threshold and then stops the comparison process to delete the two events from their respective sets. For example, event $e_{i}$ from $E_{1}$ is matched with event $e_{i}$ from $E_{2}$. The match is stopped and $e_{i}$ from $E_{1}$ and $e_{i}$ from $E_{2}$ are deleted. The candidate match event quantity is $(m+n-2)$. If each iteration can find a matched event pair, the quantity of matching events decreases regularly as an arithmetic progression. Compared with AGENES, EDCoAGENES divides cluster events into two stages, within the document (single webpage) and across documents (multiple webpages). The comparison process in the first stage is similar to AGENES, and the time complexity is $O(m)^{2}$. Given that the event quantity in one document is small, the running time is short. The second stage uses a constraint to reduce match times and quantity of candidate match events to reduce running time, and the time complexity is $O\left(m \cdot \log _{2} m\right)$. In event detection in multiple webpages, EDCoAGENES is much faster than AGENES.

\subsection{Similarity measure method}

In this paper, we propose clustering co-reference event mentions and similar events by comprehensive dimension matching. This method combines syntactic structure information and literal similarity to improve clustering accuracy. We use eight dimensions to represent an event mention and use three matchers to measure the similarity of the key dimensions. In this section, we introduce the three matchers and use extended evidence theory to combine the dimension measurement results as a similarity of an event mention.

\subsubsection{Similarity measure matcher}

We introduce three matchers to compute the similarity among the time, subject, object, and activity dimensions.

1. Time value matcher

The Time value matcher is used to measure the time dimension. This matcher is adapted for identical or compatible data types. The default time value is the "date" type. If the time in which the event occurred is precise, the time can be extended to the "date-time" type. The numerical difference between two time data is the time distance, which is multiplied by a normalized factor that can obtain time dimension similarity.

$$
\operatorname{Sim}_{N u m}\left(T_{1}, T_{2}\right)=\frac{\log D}{\left|T_{1}-T_{2}\right|}
$$

$\left|T_{1}-T_{2}\right|$ is the absolute value of the time numerical difference, and $\log D$ is the normalized factor. We set $D=20$ by experiment.

2. Morpheme matcher

Morpheme refers to the independent and basic concept, such as indivisible word. This matcher is literally indivisible. The morpheme matcher combines text similarity and word order similarity for applications to Chinese phrase characters. We add larger weights to words that appear later in the word order. For example, the subject dimension value is "50 degree Jiugui liquor". The weight of "Jiugui liquor" is larger than the weight of "50 degree". In an event mention pair, $N_{a}$ and $N_{b}$ are two 
subjects that need to be compared. The word orders are $N_{a}=\left\{a_{i} \mid i=1,2, \cdots, m\right\}$ and $N_{b}=\left\{b_{j} \mid j=1,2, \cdots, n\right\}$. Thus, the similarity of $N_{a}$ and $N_{b}$ is expressed as follows:

$$
\operatorname{Sim}_{M p}\left(N_{a}, N_{b}\right)=\frac{2}{1 / \sum \frac{a_{i}}{\sum N_{a}(i)}+1 / \sum \frac{b_{j}}{\sum N_{b}(j)}}
$$

The formula $\sum \frac{a_{i}}{\sum N_{a}(i)}$ shows the comprehensive weight of the same morpheme $N_{a}$ and $N_{b}$ contained according to the location in $N_{a} \cdot \sum \frac{b_{j}}{\sum N_{b}(j)}$ shows the comprehensive weight of the same morpheme $N_{a}$ and $N_{b}$ contained according to the location in $N_{b}$. The morpheme matcher can compute the similarity of the subject and object dimensions.

\section{Semantic matcher}

Considering that accurate reasons can be obtained by comparing verbs and verb phrases by semantic similarity, we use the semantic matcher method and the Hownet semantic network architecture [20] to measure verb similarity. In the Hownet semantic network architecture, a word is composed of primitives. The activity verb itself may be the primitive or can be deconstructed into primitives. We use primitive $S_{a}=\left\{a_{i} \mid i=1,2, \cdots, m\right\}$ to denote verb $a, S_{b}=\left\{b_{j} \mid j=1,2, \cdots, n\right\}$ to denote verb $b$, and adopted two semantic matchers to compute verb similarity.

(1) Path-based semantic matcher

The path-based semantic matcher calculates the similarity between two primitives by the path length of the two primitives.

$$
\operatorname{Sim}_{P S e}\left(S_{a}, S_{b}\right)=\frac{\alpha}{\alpha+\operatorname{dist}\left(a_{i}, b_{j}\right)}
$$

In this formula $\operatorname{dist}\left(a_{i}, b_{j}\right)$ is the shortest path in Hownet, and $\alpha$ is an adjustment parameter that is the distance value when word similarity is 0.5 . The path length is inversely proportional to similarity.

(2) Depth-based semantic matcher

The depth-based semantic matcher calculates the similarity between two primitives by the depth of their common ancestor.

$$
\operatorname{Sim}_{D S e}\left(S_{a}, S_{b}\right)=\frac{2 \times \operatorname{depth}\left(a_{i}, b_{j}\right)}{\operatorname{depth}\left(a_{i}\right)+\operatorname{depth}\left(b_{j}\right)}
$$

In this formula depth $\left(a_{i}, b_{j}\right)$ is the whole depth of the common ancestor of primitives in Hownet, depth $\left(a_{i}\right)$ and $\operatorname{depth}\left(b_{j}\right)$ denote the depth of each primitive with a common ancestor.

The following is the composite semantic similarity formula:

$$
\operatorname{Sim}_{S e m}=\beta_{1} \operatorname{Sim}_{P s e}\left(S_{a}, S_{b}\right)+\beta_{2} \operatorname{Sim}_{D s e}\left(S_{a}, S_{b}\right)
$$

In this formula $\beta_{1}+\beta_{2}=1$ and we set $\beta_{1}=0.55, \beta_{2}=$ 0.45 by experiment.
We use the maximum primitive similarity formula to calculate the similarity of two verbs:

$$
\operatorname{Sim}_{S e m}(a, b)=\max _{i=1,2, \cdots, m, j=1,2, \cdots, n}\left|\operatorname{Sim}_{S e m}\left(S_{a}, S_{b}\right)\right|
$$

\subsubsection{Use of extended evidence theory to combine} dimension matching

After obtaining the similarity of dimension value, we need to find a model to combine the dimension results as the total similarity of an event mention. Evidence theory [21] combines some evidence to obtain an objective and comprehensive result such that the model can be used to calculate the fusion result of multiple objects. In the evidence theory model, the recognition framework $\Theta$ contains all subsets, and the set of all subsets is presented as $2^{\Theta}$. The evidence theory model defines the probability distribution function as the map $2^{\Theta} \rightarrow[0,1]$, which is based on $2^{\Theta}$. The probability distribution function satisfies the conditions of $m(\phi)=0$ and $\sum_{A \in 2^{\Theta}} m(A)=1$. In the conditions, $\emptyset$ is null and $A$ is any subset. The fusion formula of the evidence theory model is as follows:

$$
m(A)=\frac{\sum_{\cap A_{i}=A} \Pi_{1 \leq i \leq n} m_{i}\left(A_{i}\right)}{1-K}, K=\sum_{\cap A_{i}=\emptyset} \Pi_{1 \leq i \leq n} m_{i}\left(A_{i}\right)
$$

In this formula, we observe that each dimension provides the same contribution degree to the final result. However, the evidence characteristics of different dimensions are not the same. For example, we extract event mentions in webpages that are reported in the same day. In this situation, the similarity of the subject dimension is more important than the time dimension. To solve this problem and obtain a more accurate value for the similarity of the event mention, we extend the evidence theory model by allocating dynamic weight factor $W_{i}$ for each dimension. We add a dynamic weight factor in each probability distribution function, and the fusion formula of extended evidence theory model is as follows:

$$
m(A)=\frac{\sum_{\cap A_{i}=A} \Pi_{1 \leq i \leq n} W_{i}\left[m_{i}\left(A_{i}\right)\right]}{\sum_{\cap A_{i} \neq \emptyset} \Pi_{1 \leq i \leq n} W_{i}\left[m_{i}\left(A_{i}\right)\right]}
$$

In this formula, $W_{i}$ is the ability weight factor for every dimension, and $\sum W_{i}=1$. The following dimension-matching algorithm shows how to calculate comprehensive dimension similarity.

In Algorithm 3, Line 1 compares the dimension similarity of a given event mention pair. Lines 2 to 5 compute the similarity of four dimensions as different similarity evidences, and Line 6 uses extended evidence theory to compute the comprehensive similarity of the event mention. The EDCoAGENES cluster method uses extended evidence theory to allocate dynamic weight and this method is more accurate than static weight. We will compare EDCoAGENES with other cluster methods in the experiment. 


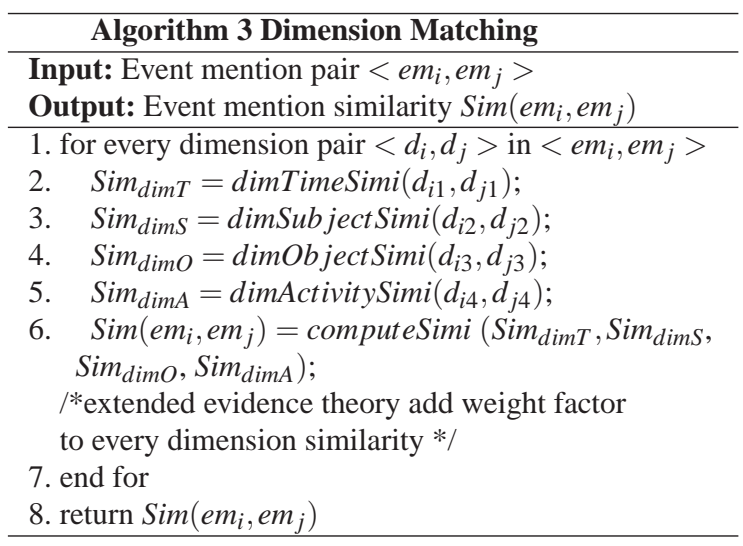

\section{Experiment}

\subsection{Experiment dataset}

We extracted 9,000 event mentions in the food safety, phone, and computer fields as three experiment datasets. These event mentions were extracted from reports in news webpages during one week (from Aug 8, 2012 to Aug 15, 2012). We labeled the event mention that contains target entities and used eight dimensions to represent the event mention.

\subsection{Experiment evaluation}

The event detection discovered event clusters that contained many co-reference event mentions. To evaluate efficiency of the clustering method, we used an information retrieval evaluation method and divided the cluster results into four sets.

$\mathrm{A}=$ True Positives (event mentions that are clustered in a cluster is correct)

$\mathrm{B}=$ False Negatives (event mentions that are not clustered in a cluster is incorrect)

$\mathrm{C}=$ False Positives (event mentions that are clustered in a cluster is incorrect)

$\mathrm{D}=$ True Negatives (event mentions that are not clustered in a cluster is correct)

The precision, recall, and F-measure are calculated using the Equations (9), (10) and (11).

$$
\begin{gathered}
\text { Recall }=\frac{|A|}{|A|+|B|} \\
\text { Precission }=\frac{|A|}{|A|+|C|} \\
F-\text { measure }=\frac{2 \times \text { Recall } \times \text { Precission }}{\text { Recall }+ \text { Precission }}
\end{gathered}
$$

"Recall" evaluates the cover degree of correct clustering, "precision" evaluates the soundness of the

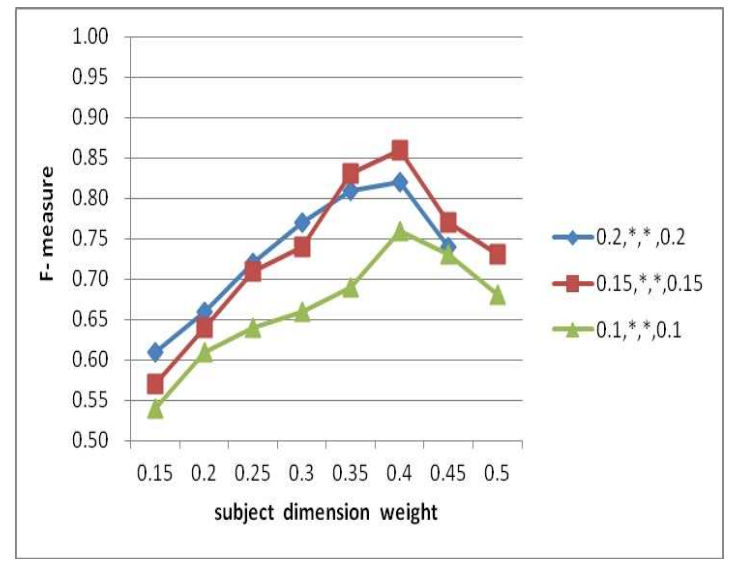

Fig. 6: Assessment of dynamic weight on four dimensions

clustering, and " $F-$ measure" is the comprehensive evaluation.

According to the comprehensive dimension matching and co-occurrence constraint proposed in this paper, we tested the effectiveness of event detection in the following experimental aspects: (1) assessment of the extended evidence theory model to allocate dynamic dimension weights for clusters; (2) use of the co-occurrence constraint to reduce running times for event detection in multiple webpages; (3) comparison of the effectiveness of different event detection methods.

\subsection{Experimental result and analysis}

1. We used the extended evidence theory model to allocate weights and acquire the best weight distribution.

We used the extended evidence theory model to allocate dimension weight and obtain different cluster results. Important dimension needs to be allocated with a larger weight to obtain better weight allocation.

Fig. 6 shows some allocation plans by experiments. First, we fixed the time and activity dimensions by allocating different weights and adjusting the rest weight on the subject and object dimensions. $(0.2, *, *, 0.2)$ means that the weight of time and activity are both fixed to 0.2 , and the remaining 0.6 weight will be distributed to the subject and object dimensions. Fig. 6 shows that $(0.15,0.4,0.3,0.15)$ is the best weight allocation because the F-measure reaches the highest point. To compare the dynamic weight, we clustered the static weight $(0.25$, $0.25,0.25,0.25$ ), and the F-measure is only 0.67 .

2. Evaluation of the effect of co-occurrence constraint in restricting the cluster process for event detection in multiple webpage.

Fig. 7 shows the running time of two cluster methods on different event mention datasets. No noticeable difference was observed in the running time in small datasets, such as those that contain only 100 and 200 


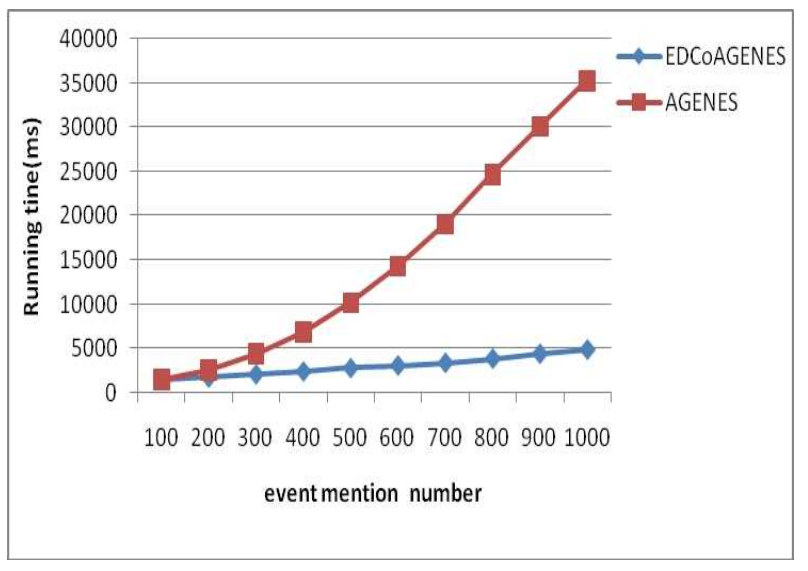

Fig. 7: Comparsion of running times of AGENES and EDCoAGENES

\begin{tabular}{|c|c|c|c|c|}
\hline \multirow{3}{*}{ nethodd } & \multicolumn{4}{|c|}{ event nention anount } \\
\cline { 2 - 5 } & 500 & 1000 & 1500 & 2000 \\
\cline { 2 - 5 } & \multicolumn{4}{|c|}{ conpare tines } \\
\hline AGEVES & 124750 & 499500 & 1124250 & 1999000 \\
\hline \multirow{2}{*}{ EDCodGENES } & 17385 & 36735 & 57685 & 80235 \\
$(12250+5135)$ & $(24500+12235)$ & $(36750+20935)$ & $(49000+31235)$ \\
\hline
\end{tabular}

Fig. 8: Compare match times with AGENES and EDCoAGENES

event mentions. In larger datasets, EDCoAGENES uses a co-occurrence constraint that can significantly reduce running time. We propose the EDCoAGENES method for event detection in multiple webpages to reduce event match times and quantity of candidate matches events. Fig. 8 presents the different match times of the two cluster methods.

We chose four different event mention datasets to compare match times. In the EDCoAGENES line, $(12,250+5,135)$ denotes that match times in a single webpage are added with match times between web pages, and 17,385 denote the sum of two parts. Fig.8 shows that EDCoAGENES, which uses two-stage event detection and a co-occurrence constraint, can reduce match times significantly.

3. We evaluated the effectiveness of using different cluster methods for event detection in multiple web pages. We compared the K-means, AGENES, and EDCoAGENES methods to detect events on food safety and phone datasets. The recall, precision, and F-measure of event detection are shown in Fig.9 and Fig. 10.

We find that the EDCoAGENES method, which uses extended evidence theory to combine dimension-matching results and allocate dynamic dimension weights, has the higher recall, precision, and F-measure than other cluster methods (Figs. 9 and 10). To prove that this cluster method is not restricted to a special field, the experiment was conducted by using a different

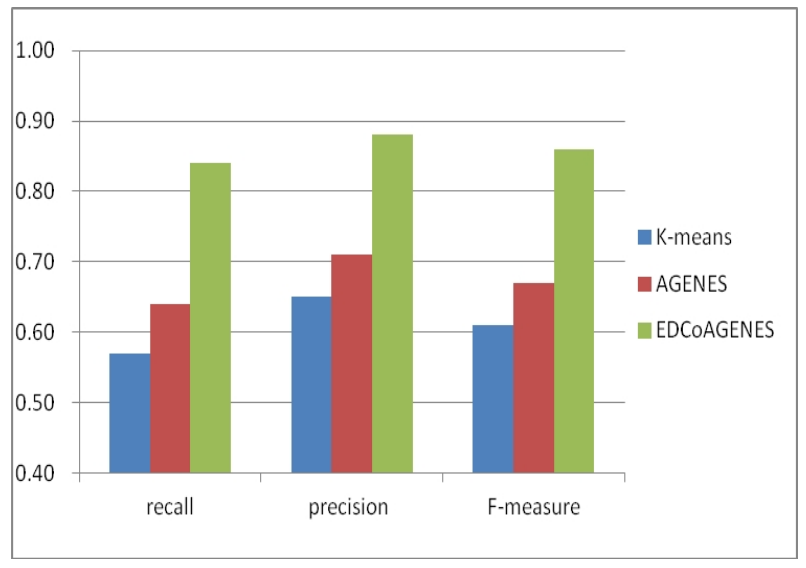

Fig. 9: Recall, precision, and F-measure of three methods on food safety dataset

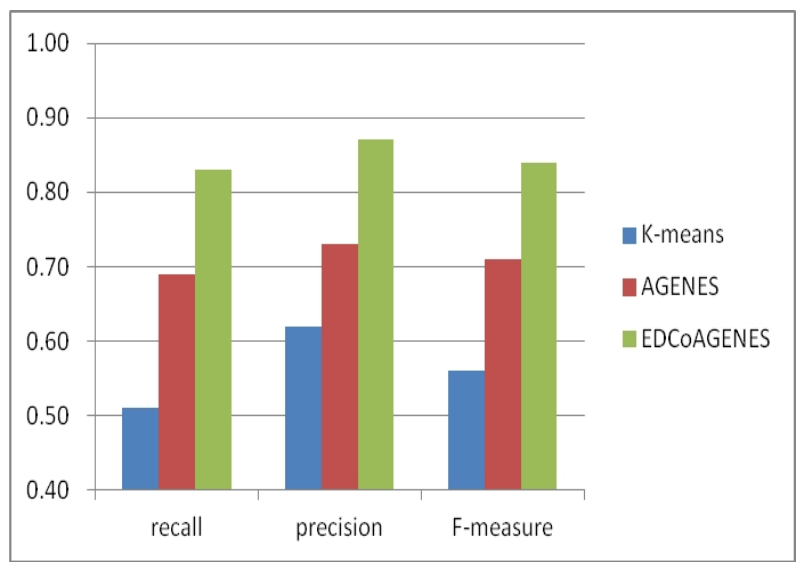

Fig. 10: Recall, precision and F-measure of three methods on phone dataset

field dataset. The results show that EDCoAGENES is better than K-means or AGENES.

\section{Conclusion}

In this paper, we have proposed a method for detecting events in multiple webpages, namely, EDCoAGENES, which is based on comprehensive dimension matching and co-occurrence constraint theory. We detected single webpage events and found co-occurrence events in every single webpage. We presented an event co-occurrence constraint based on single webpage detection to reduce event match times and quantity of candidate matches events in multiple webpages. In event clustering process, we used eight dimensions to represent event mentions and compare the similarity of key dimensions. We used extended evidence theory to allocate the dynamic weight for different dimensions and combined the comprehensive 
results as the similarity of event mentions. The experiment results demonstrate that this method can quickly detect various events in multiple webpages and can effectively reduce the number of co-reference events.

\section{Acknowledgement}

This work is supported by the National Key Technologies R\&D Program (No.2012BAH54F01), Shandong Province Independent Innovation Major Special Project (No.2013CXC30201), the Natural Science Foundation of China (No.61303005) and the Shandong Distinguished Middle-aged and Young Scientist Encouragement and Reward Foundation (No.BS2012DX015).

\section{References}

[1] C. Y. Zhang, X. G. Hong, Z. H. Peng, Journal of Software, 23, 2612-2627 (2012).

[2] C. J. Fillmore, Quademi di Semantica, 222-253, (1986).

[3] Bo Zhang and Zhicai Juan, Modeling User Equilibrium and the Day-to-day Traffic Evolution based on Cumulative Prospect Theory, Information Science Letters, 2, 9-12 (2013).

[4] D. S. Anish, J. Alpa and C. Yu, Proc of the 4th International Conference on Web Search and Web Data Mining, Hong Kong, China, 207-216 (2011).

[5] J. Yao, B. Cui, Y. Huang and Y. Zhou, World Wide Web, 15, 171-195 (2012).

[6] W. S. Pan, S. Z. Chen, Z. Y Feng, Applied Mathematics \& Information Sciences, 7, 675-681 (2013).

[7] M. K. Agarwal, K. Ramamritham and M. Bhide, Proceedings of the VLDB Endowment, 5, 980-991 (2012).

[8] R. Yan, Y. Li, Y. Zhang and X. Li, Information Retrieval Technology, 490-501, (2010).

[9] D. Shan, W. X. Zhao, R. Chen, B. Shu, Z. Wang, J. Yao, H. Yan and X. Li, Proceedings of the 18th ACM SIGKDD International Conference on Knowledge Discovery and Data Mining, 18, 1564-1567 (2012).

[10] W. X. Zhao, R. Chen, K. Fan, H. Yan, and X. Li, Proceeding of the 50th Annual Meeting of the Association for Computational Linguistics Jeju, 50, 43-47 (2012).

[11] M. Naughton, N. Stokes and J. Carthy, Information retrieval, 13, 132-156 (2010).

[12] B. Jiang, M.-x. Zhu, and J.-1. Wang, Journal of Computers, 8, 85-90 (2013).

[13] D. A. Smith, Proceeding of the 25th Annual International ACM SIGIR Conference on Research and Development in Information Retrieval, 25, 73-80 (2002).

[14] Z. Lei, J. Liao, D. Li and L. Wu, Proceedings of the 4th International Conference on Intelligent Computing, 4, 872879 (2008).

[15] T. Xiëski, A. Nowak-Brzeziska and A. Wakulicz-Deja, Proceeding of the 8th International Conference, 8, 142-149 (1956).

[16] L. Zheng, L. Li, W. Hong and T. Li, Expert Systems with Applications, 40, 2127-2136 (2013).
[17] Z. M. Zhong and Z. T. Liu, Pattern Recognition and Artificial Intelligence, 23, 307-313 (2010).

[18] C. Li, Z. Xu and T. Luo, Pattern Recognition Letters, 34, 155-162 (2013).

[19] S. Salicone, IEEE Instrumentation \& Measurement Magazine, 16, 18-23 (2013).

[20] http://www.keenage.com/.

[21] P. S. Medeiros Dos Santos and G. H. Travassos, Electronic Notes in Theoretical Computer Science (ENTCS), 292, 95118 (2013).

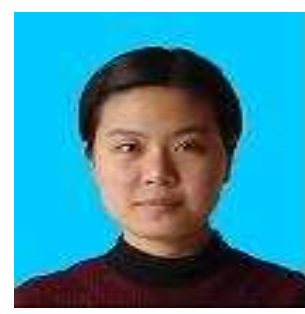

Yuanzi Xu received the Bachelor's degree from Zhengzhou University in 2006, and the Master's degree from Shandong University in 2009. She is currently a Ph.D candidate in Shandong University of computer science and technology. Her research interests are in the areas of Web information integration and event detection.

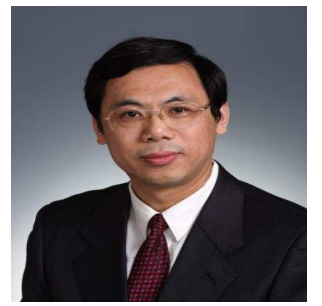

Qingzhong Li received the Master's degree from Shandong University in 1989, and the Ph.D from the Institute of Computing Technology Chinese Academy of Sciences in 2000. He is currently a professor in Shandong University. His research interests are in the areas of Web information integration and large-scale network data management.

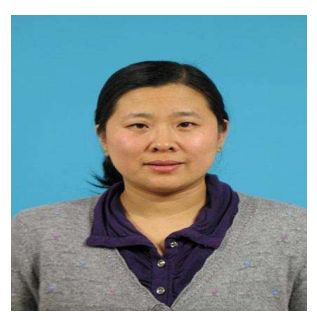

Zhongmin Yan received the Ph.D degree from Shandong University in 2010. She is currently an associate professor in Shandong University. Her research interests are in the areas of Web information integration and Web data management.

Wei Wang received

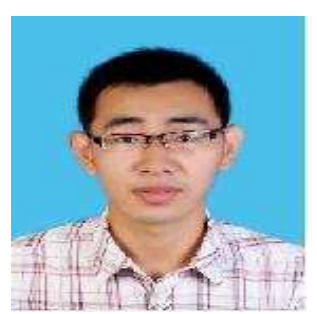
the Bachelor's degree from Shandong University in 2012. He is currently a master candidate in Shandong University. His research interests are Web information integration and event detection. 